\title{
Fine Structure of $\mathrm{Zr}_{80} \mathrm{Pt}_{20}$ Amorphous Alloy Determined from Anomalous $\mathrm{X}$-ray Scattering (AXS) Data by Applying Reverse Monte-Carlo (RMC) Simulation Method
}

\author{
T. Kawamata, T. Muto and K. Sugiyama \\ Institute for Materials Research, Tohoku University, Sendai 980-8577, Japan
}

The fine structure of a $\mathrm{Zr}_{80} \mathrm{Pt}_{20}$ amorphous alloy was evaluated by anomalous X-ray scattering (AXS) coupled with reverse Monte Carlo (RMC) simulation. Featureless short-range ordered structures were preferentially formed by the Zr component, similar to those described by dense random packing (DRP), while the Pt atoms adopted a somewhat different structure. The Pt atoms adopted Zr-rich coordination and an icosahedral local atomic arrangement, in unique chemical short-range ordering (CSRO) as well as topological short-range ordering (TSRO). The present AXS-RMC analysis also suggested that the pre-peak signal at $17 \mathrm{~nm}^{-1}$ in the X-ray diffraction profile was largely attributed to the correlation between the Pt-Pt pair with a separation of approximately $0.4-0.5 \mathrm{~nm}$. The geometrical and chemical features of the common neighbors of the middle-range Pt-Pt pairs indicate the unique CSRO and TSRO, where both chemical and density fluctuations were observed in two distinct regions: a Zr-rich region with high packing density and a Pt-rich region with low packing density.

[doi:10.2320/matertrans.MT-M2020246]

(Received August 3, 2020; Accepted October 5, 2020; Published December 4, 2020)

Keywords: metallic glass, anomalous X-ray scattering, reverse Monte-Carlo simulation, amorphous alloy, structural study, medium range ordering

\section{Introduction}

Atomic ordering in both the short-range region and the middle-range region in amorphous alloys is known to be closely correlated with unique material properties. ${ }^{1-4)}$ Nevertheless, atomic ordering in amorphous alloys is poorly understood and suitable methods of describing the subtle differences in the ordering are still far from complete.

As an example, the icosahedral (and icosahedral-like) local atomic arrangement is suggested to be one of the specific topological short range orderings (TSROs) in Zr-based amorphous alloys. ${ }^{6}$ ) Researchers propose that such TSRO is associated with solute-centered, solvent-shell type chemical short-range ordering (CSRO). ${ }^{7)}$ In contrast with short-range ordering (SRO), the so-called middle-range ordering (MRO), which involves atomic ordering reaching the next shell around the first nearest neighbor region, is yet to be fully elucidated and visualized.

Experimental evidence of MRO has been reported based on the occasional observation of a pre-peak signal in the $\mathrm{X}$ ray diffraction (XRD) and neutron diffraction (ND) patterns of several amorphous alloys. ${ }^{8-11)}$ Recent studies of $\mathrm{Zr}-\mathrm{Pt}$ binary amorphous alloys ${ }^{12-14)}$ strongly suggest that the prepeak signal may provide information about the characteristic atomic ordering of amorphous alloys through a relatively less complex chemical approach. Structural studies of $\mathrm{Zr}_{80} \mathrm{Pt}_{20}$ amorphous alloys also suggest that the icosahedral (-like) local atomic arrangement is the dominant TSRO and the pre-peak arises from the enhanced middle range $\mathrm{Pt}-\mathrm{Pt}$ correlation. ${ }^{7,14-17)}$ However, no quantitative information has yet been obtained with respect to the relationship between the icosahedral TSRO and MRO or the specific atomic arrangements that contribute to enhancing the middle-range Pt-Pt correlation.

The main purpose of this work is to report the fine structure of a $\mathrm{Zr}_{80} \mathrm{Pt}_{20}$ amorphous alloy, determined from anomalous X-ray scattering (AXS) data ${ }^{17)}$ by applying the reverse Monte-Carlo (RMC) simulation method, from which a method for describing the atomic ordering in the middlerange region is proposed by introducing a new approach for distribution of the Bernal polyhedral units.

\section{Methodology of AXS-RMC Analysis}

The AXS data for the $\mathrm{Zr}_{80} \mathrm{Pt}_{20}$ amorphous alloy were taken from our previous paper, ${ }^{17}$ and the procedure for the RMC simulation employed in this work is identical to that described in our previous works. ${ }^{5,18,19)}$ Only the essential points are given below for convenience of discussion. When the incident energy for AXS is set close below the absorption edge, $E_{a b s}$, using $\mathrm{Pt}$ as an example in this case, anomalous dispersion becomes significant. The resulting variation in the signal intensity, $\Delta i_{P t}\left(Q, E_{1}, E_{2}\right)$, is attributed to a change in the real part of the anomalous dispersion terms of $\mathrm{Pt}$.

$$
\begin{aligned}
& \Delta i_{P t}\left(Q, E_{1}, E_{2}\right) \\
& \equiv \frac{\left\{I\left(Q, E_{1}\right)-\left\langle f^{2}\left(Q, E_{1}\right)\right\rangle\right\}-\left\{I\left(Q, E_{2}\right)-\left\langle f^{2}\left(Q, E_{2}\right)\right\rangle\right\}}{c_{P t}\left\{f_{P t}^{\prime}\left(E_{1}\right)-f_{P t}^{\prime}\left(E_{2}\right)\right\} W\left(Q, E_{1}, E_{2}\right)} \\
& =\frac{c_{P t} \Re\left\{f_{P t}\left(Q, E_{1}\right)+f_{P t}\left(Q, E_{2}\right)\right\}}{W\left(Q, E_{1}, E_{2}\right)}\left(a_{P t P t}(Q)-1\right) \\
& +\frac{c_{Z r} \Re\left\{f_{Z r}\left(Q, E_{1}\right)+f_{Z r}\left(Q, E_{2}\right)\right\}}{W\left(Q, E_{1}, E_{2}\right)}\left(a_{P t Z r}(Q)-1\right) \\
& \quad W\left(Q, E_{1}, E_{2}\right)=\sum_{k=1}^{2} c_{k} \Re\left\{f_{k}\left(Q, E_{1}\right)+f_{k}\left(Q, E_{2}\right)\right\}
\end{aligned}
$$

Here, $Q$ and $E$ are the wave vector and the incident X-ray energy, respectively, and $c_{\mathrm{i}}$ is the atomic fraction. The atomic scattering factor of the $i$-th element is given by the equation: $f_{i}(Q . E)=f_{i}^{0}(Q)+f_{i}^{\prime}(E)+i f_{i}^{\prime \prime}(E)$, where $f^{0}(Q)$ is the scattering factor of the atom at an energy sufficiently away from the absorption edge, and $f^{\prime}(E)$ and $f^{\prime \prime}(E)$ are the real and imaginary parts of the anomalous dispersion terms, ${ }^{20)}$ respectively. Equation (2) includes two functions: $I(Q . E)$ and $W\left(Q . E_{1}, E_{2}\right)$. The function $I(Q . E)$ is the coherent X-ray scattering intensity, obtained by ordinary analysis coupled 
with the generalized Krough-Moe-Norman method. ${ }^{21)}$ The function $W\left(Q, E_{1}, E_{2}\right)$ is calculated using eq. (2), where the symbol $\Re\{\}$ denotes the real part of the values in the brackets. The value of $\Delta i_{P t}\left(Q, E_{1}, E_{2}\right)$ is a function of the two partial structure factors, $a_{P t P t}(Q)$ and $a_{P t Z r}(Q)$. Similarly, $\Delta i_{Z r}\left(Q, E_{1}, E_{2}\right)$ measured at the $\operatorname{Zr} K$ absorption edge contains contributions from $a_{Z r Z r}(Q)$ and $a_{P t Z r}(Q)$. Fouriertransforms of the functions $\Delta i_{P t}\left(Q, E_{1}, E_{2}\right)$ and $\Delta i_{Z r}\left(Q, E_{1}, E_{2}\right)$ using the average number density $\left(\rho_{0}\right)$ of the system readily yields the environmental radial distribution functions (RDFs) $4 \pi r^{2} \rho_{\mathrm{Pt}}(r)$ and $4 \pi r^{2} \rho_{\mathrm{Zr}}(r)$, respectively.

The detailed parameters for the RMC simulation ${ }^{22)}$ are summarized as follows. The simulation was initiated with a model comprising 25000 atoms (Zr: 20000 and Pt: 5000) for the dense random packing (DRP) structure in a cubic hyper-cell with a unit size of $L=8.102 \mathrm{~nm}$. The number of atoms and cell size were selected for the model to meet the chemical composition as well as the measured density $\left(8.74 \mathrm{Mg} / \mathrm{m}^{3}\right)$ of the $\mathrm{Zr}_{80} \mathrm{Pt}_{20}$ sample. The atomic positions in the DRP structure were obtained using the algorithm proposed by Clarke et al. ${ }^{23)}$ by employing the Goldschmidt radii $\left.(\mathrm{Zr}: 1.60 \mathrm{~nm}, \mathrm{Pt}: 1.38 \mathrm{~nm}){ }^{24}\right)$ Through the present RMC simulation, the partial distribution functions for each pair of constituents and the corresponding structural factors could be readily calculated, where this structural information is very useful for discussing the SRO and MRO observed for amorphous $\mathrm{Zr}_{80} \mathrm{Pt}_{20}$.

\section{Results}

Figure 1 shows the experimental interference functions, $Q i(Q), Q \Delta i_{P t}(Q)$, and $Q \Delta i_{Z r}(Q)$, as well as those determined from the AXS data using the RMC simulation method (hereafter referred to as AXS-RMC). The AXS-RMC simulation results reproduced the experimental structural functions well, and the atomic positions obtained from the

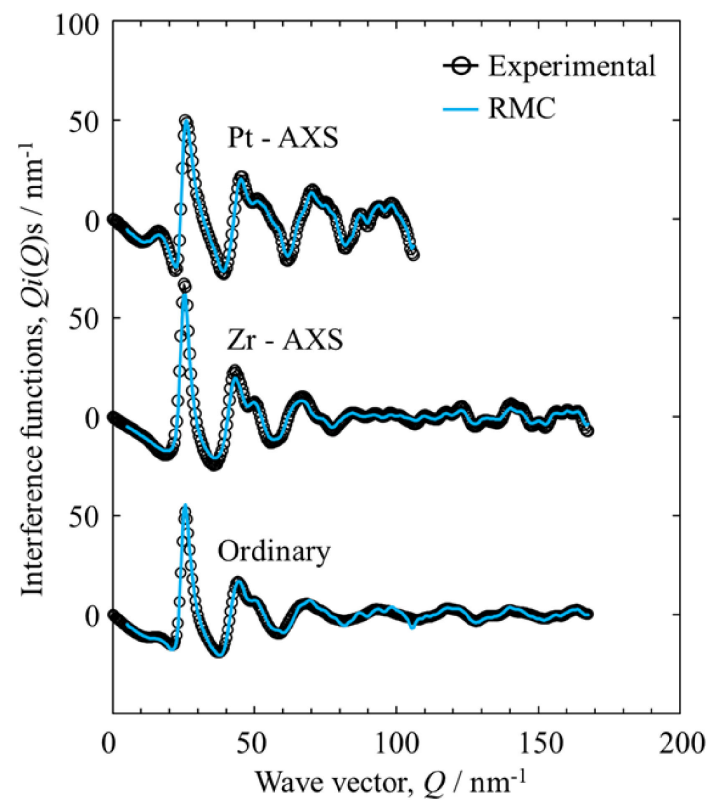

Fig. 1 Experimental interference functions, $Q \Delta i_{P t}(Q), Q \Delta i_{Z r}(Q)$, and $Q i(Q)$, and corresponding values obtained from RMC simulation.

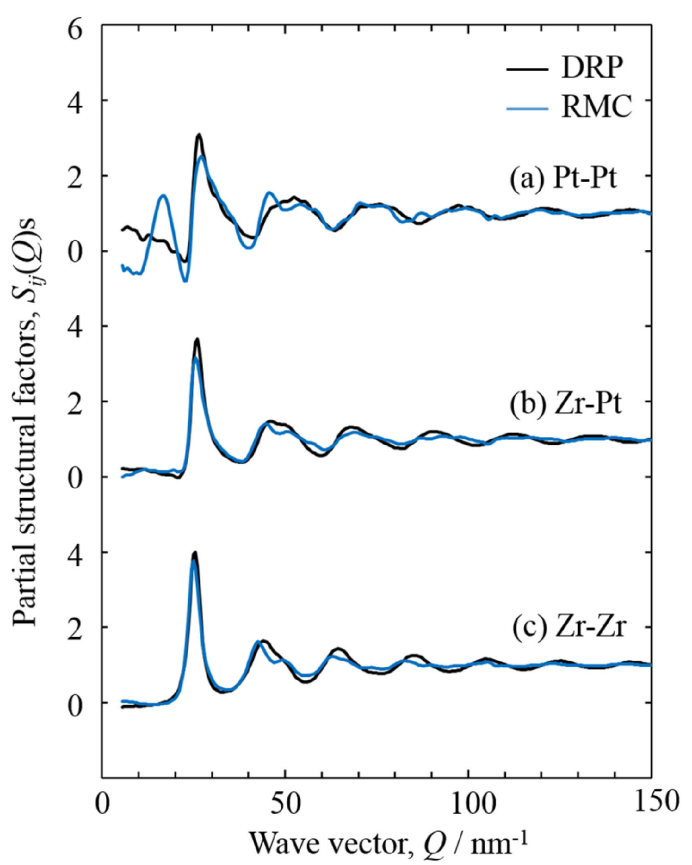

Fig. 2 Partial structure factors, $S_{P t P t}(Q), S_{Z r Z r}(Q)$, and $S_{P t Z r}(Q)$ calculated from the RMC simulation and using DRP structural model.

present AXS-RMC simulation seem to be much closer to the atomic arrangements of amorphous $\mathrm{Zr}_{80} \mathrm{Pt}_{20}$.

Notably, a pre-peak signal was clearly observed at approximately $Q=17 \mathrm{~nm}^{-1}$ for the experimental functions $Q i(Q)$ and $Q \Delta i_{P t}(Q)$, but not for the function $Q \Delta i_{Z r}(Q)$. This observation suggests that the pre-peak signal is associated with the Pt-Pt interaction in the middle-range ordering. ${ }^{17)}$

Figure 2 shows three partial structure factors, $S_{i j}(Q) \mathrm{s}$, of the $\mathrm{Zr}_{80} \mathrm{Pt}_{20}$ amorphous alloy in the so-called Faber-Ziman (FZ) form obtained from the present AXS-RMC simulation and those of the DRP structure model. Because the pre-peak signal at $17 \mathrm{~nm}^{-1}$ was clearly observed in $S_{P t P t}(Q)_{\text {AXS-RMC, }}$ the middle range ordering structure of the $\mathrm{Zr}_{80} \mathrm{Pt}_{20}$ amorphous alloy is attributed to the $\mathrm{Pt}-\mathrm{Pt}$ pair correlation and the corresponding chemical fluctuation.

Figure 3 shows the partial pair distribution functions, $g_{i j}(r) \mathrm{s}$, determined from the AXS-RMC results, as well as those determined from the DRP structure model. The vertical dotted lines indicate the nearest neighbor atomic distances for the Pt-Pt, Zr-Pt, and Zr-Zr pairs (Pt-Pt: $0.276 \mathrm{~nm}, \mathrm{Zr}-\mathrm{Pt}$ : $0.298 \mathrm{~nm}, \mathrm{Zr}-\mathrm{Zr}$ : $0.320 \mathrm{~nm}$ ), estimated from the Goldschmidt radii. ${ }^{24)}$ The first peaks of the partial pair distribution functions, $g_{P t P t}(r)_{\mathrm{AXS}-\mathrm{RMC}}$ and $g_{\mathrm{ZrZr}}(r)_{\mathrm{AXS}-\mathrm{RMC}}$, were found at a distance that is just twice the Goldschmidt radii. On the other hand, the first peak corresponding to $g_{Z r P t}(r)_{\mathrm{AXS}-\mathrm{RMC}}$ had an apparent maximum at a distance shorter than that estimated using the Goldschmidt radii. The upper limit of nearest neighbor region for each atomic pairs used in calculation of SRO parameters and common neighbor analysis (CNA) were set as the near of the minimum $\mathrm{g}(r)$ as follows, $\mathrm{Zr}-\mathrm{Zr}$ : $0.420 \mathrm{~nm}, \mathrm{Zr}-\mathrm{Pt}$ : $0.400 \mathrm{~nm}$ and Pt-Pt: $0.380 \mathrm{~nm}$.

For all three $g_{i j}(r)_{\text {AXS-RMC }}$ partial pair distribution functions, a second correlation peak consisting of two humps was observed at a distance of $0.45-0.65 \mathrm{~nm}$. Importantly, the 


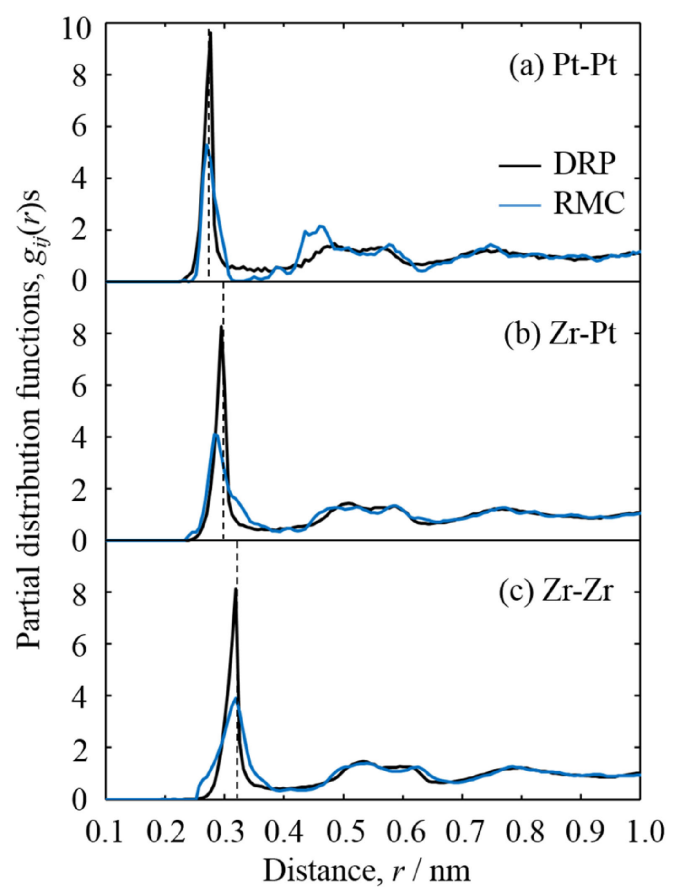

Fig. 3 Partial pair distribution functions, $g_{i j}(Q)$ s, calculated from 3-D structural model obtained by RMC simulation. The corresponding functions for the DRP structure are also illustrated for comparison.

partial pair distribution function $g_{\mathrm{PtPt}}(r)_{\mathrm{AXS}-\mathrm{RMC}}$ indicates significant enlargement at the shorter side of the second correlation peak. This unique correlation provides key information on the MRO developed in the present $\mathrm{Zr}_{80} \mathrm{Pt}_{20}$ amorphous alloy.

\section{Discussion}

\subsection{Short range ordering}

Table 1 lists the structural parameters, including the average atomic distance and the coordination numbers for the nearest-neighbor region, calculated from the threedimensional atomic models obtained from the AXS-RMC simulation results. The structural parameters determined from the DRP model are also presented in Table 1 in the round brackets.

Examination of the listed parameters from AXS-RMC readily suggests chemical affinity between the first-nearest $\mathrm{Pt}-\mathrm{Zr}$ pair, as well as inhomogeneous distribution of Pt. As an example, the maximum of the first peak of the $\mathrm{Pt}-\mathrm{Zr}$ pair was found at $0.282 \mathrm{~nm}$, where the distance is shorter than that of the DRP structure. This is likely to arise from the chemically strong bond associated with the small mixing enthalpy of $\mathrm{Pt}$ and $\mathrm{Zr}^{25}$ ) On the other hand, the average atomic distance, $r_{i j}$, and the average coordination number, $Z_{i j}$, of $\mathrm{Zr}$ were not significantly different from those of the DRP structure. This again implies that the averaged local structure of $\mathrm{Zr}$ surrounded by the solvent is well approximated by the DRP structure.

The Voronoi tessellation ${ }^{26)}$ is a well-known and useful method of evaluating the fine topological features around specific components of amorphous structural models. We analyzed the distribution of the Voronoi polyhedral units of the AXS-RMC and DRP structural models in a previous report. ${ }^{17)}$ The distribution of the Voronoi polyhedral units around the $\mathrm{Zr}$ atoms agrees well with the DRP structure, while some differences can be found in the distribution of the Pt atoms. This contrast suggests that the TSRO around the $\mathrm{Pt}$ atoms is influenced by chemical interactions. The icosahedral and icosahedron-like coordination indicated by

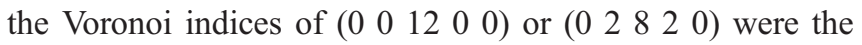
dominant TSRO in the Voronoi polyhedra distribution for the Pt atoms. This result indicates that the Pt center adopts icosahedral short-range ordering in the $\mathrm{Zr}_{80} \mathrm{Pt}_{20}$ amorphous structure as one of the most important TSROs.

It should be noted that the topological preference of the $\mathrm{Pt}$ center for icosahedral(-like) SRO can be explained by using the ratio, $r^{*}$, of the first-nearest $\mathrm{Pt}-\mathrm{Zr}$ and $\mathrm{Zr}-\mathrm{Zr}$ distances, which corresponds to the ratio of the distance from the center to the vertex and that to the edge in the Pt-centered icosahedrons. The calculated ratio $r^{*}$ was $0.951=0.311 /$ 0.327 , which clearly coincides with $r_{\text {ico }}{ }^{*}=0.951$ for the ideal icosahedron.

\subsection{Common neighbor analysis and middle-range order- ing with structural inhomogeneity}

Analysis of the SRO in the first nearest-neighbor region is useful for understanding the fundamental structural features of amorphous alloys. However, it is sometimes necessary to conduct further analysis with respect to the second nearestneighbor region. The present $\mathrm{Zr}_{80} \mathrm{Pt}_{20}$ amorphous alloy could be included in this category because the partial pair distribution function of the Pt-Pt pair indicates a significant enlargement at approximately $0.45 \mathrm{~nm}$ in the second

Table 1 Average atomic distance: $r_{i j}$, first peak maximum for $g_{i j}(r): p_{i j}$, average coordination number: $Z_{i j}$, chemical occupancy of coordinated atoms, average atomic size ratio, $R^{*}$, from RMC simulation. The $R^{*}$ values for the DRP structure are also presented in round brackets for comparison.

\begin{tabular}{ccccccc}
\hline$i$ & $j$ & $r_{i j}[\mathrm{~nm}]$ & $p_{i j}[\mathrm{~nm}]$ & $Z_{i j}$ & Occup. [\%] & $R^{*}$ \\
\hline \multirow{2}{*}{$\mathrm{Zr}$} & $\mathrm{Zr}$ & $0.327(0.328)$ & $0.319(0.319)$ & $11.3(11.0)$ & $82.5(82.0)$ & $1.025(1.025)$ \\
& $\mathrm{Pt}$ & $0.311(0.309)$ & $0.282(0.299)$ & $2.4(2.4)$ & $17.5(18.0)$ & \\
\hline
\end{tabular}

$13.7(13.0)$

\begin{tabular}{ccccccc}
\hline \multirow{3}{*}{$\mathrm{Pt}$} & $\mathrm{Zr}$ & $0.311(0.309)$ & $0.282(0.299)$ & $9.5(9.6)$ & $86.4(82.1)$ & $0.879(0.862)$ \\
& $\mathrm{Pt}$ & $0.284(0.289)$ & $0.270(0.276)$ & $1.5(2.1)$ & $13.6(17.9)$ & \\
\hline \multicolumn{6}{c}{$11.0(11.7)$}
\end{tabular}




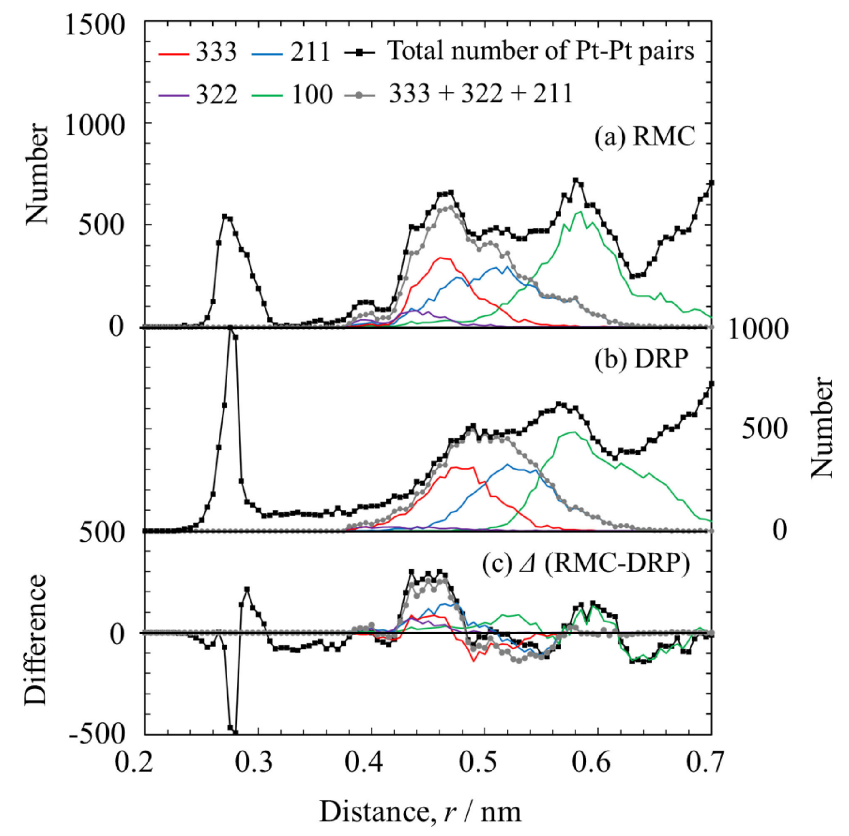

Fig. 4 Results of CNA for middle-range Pt-Pt pair from RMC simulation (a), corresponding data for DRP structure (b), and the difference between both sets of values (c).

correlation region, as shown in Fig. 3. To extend the analysis to the second nearest-neighbor region, the $\mathrm{CNA}^{27,28)}$ was employed.

An interesting pair correlation was selected for CNA, where the paired atoms were specified as the root-pair atoms. The CNA procedure ranks the atomic configuration for the root-pair atoms by using CNA indices of three numbers, $\left[\begin{array}{lll}n_{1} & n_{2} & n_{3}\end{array}\right]_{\mathrm{CN}}$. Here, $n_{1}$ is the number of neighbors common to the root-pair atoms, $n_{2}$ is the number of connections between the common neighbors, and $n_{3}$ is the longest continuous number of connections between common neighbors.

Figure 4 shows the results of CNA for the Pt-Pt correlation associated with the second peak region of the partial $g_{P t P t}(r)_{\mathrm{AXS}-\mathrm{RMC}}$ and $g_{P t P t}(r)_{\mathrm{DRP}}$. Schematic diagrams of the typical common neighbor connections found in this region, i.e., $[333]_{\mathrm{CN}},[322]_{\mathrm{CN}}$, and $[211]_{\mathrm{CN}}$, are illustrated in Fig. 5. The numbers, the average distance of the root-pairs, and the elemental fraction of the common neighbors for each connection are summarized in Table 2. The root-pairs in the second nearest-neighbor region are ranked into four CNA indices, i.e., $[333]_{\mathrm{CN}},[322]_{\mathrm{CN}},[211]_{\mathrm{CN}}$, and $[100]_{\mathrm{CN}}$. The middle-range Pt-Pt correlation around $0.45 \mathrm{~nm}$ mainly consists of the connection defined by the three indices $[333]_{\mathrm{CN}},[322]_{\mathrm{CN}}$, and $[211]_{\mathrm{CN}}$. This connection is charac- (a)

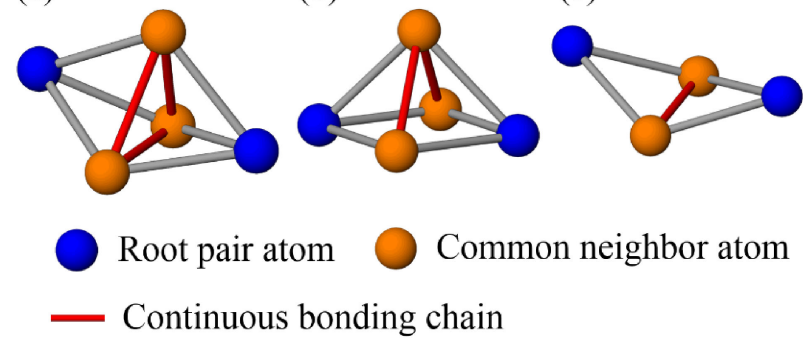

Fig. 5 Schematic diagrams of bonding between root pair atoms and common neighbor atoms with CNA index of (a) [333], (b) [322], and (c) $[211]$.

terized by a short distance and a remarkably higher population of $\mathrm{Zr}$ in the common neighbors. This result readily suggests that the second-nearest $\mathrm{Pt}-\mathrm{Pt}$ pairs, which are closely associated with the pre-peak signal, accommodate $\mathrm{Zr}$ as common neighbors to produce a Zr-rich CSRO around Pt. At the same time, there is a high preference for the $[322]_{\mathrm{CN}}$ and $[211]_{\mathrm{CN}}$ connections at $0.45 \mathrm{~nm}$ as another interesting feature observed in the present AXS-RMC results. This is discussed in the next paragraph by using a new approach employing the distribution of the Bernal polyhedral units.

\subsection{Geometrical features of MRO around Pt}

To discuss the topological features in the connection for the specific middle-range Pt-Pt ordering associated with the pre-peak signal, we introduce the distribution of the Bernal polyhedral units. ${ }^{29)}$

The connection specified by the CNA index $[333]_{\mathrm{CN}}$ indicates a structure composed of two Pt atoms as a rootpair and three atoms as common neighbors. This local structure can be considered as a unit of face-sharing tetrahedra (FST), as shown in Fig. 5. Because the tetrahedron is one of the dense packing structural units among Bernal polyhedra, the connection with the $[333]_{\mathrm{CN}}$ index corresponds to a relatively more dense packing structure. The $[333]_{\mathrm{CN}}$ connection could be further ranked by the number of $\mathrm{Zr}$ atoms among the three neighbors $\left(\mathrm{Zr}_{3}, \mathrm{Zr}_{2} \mathrm{Pt}, \mathrm{Zr}_{1} \mathrm{Pt}_{2}\right.$, and $\mathrm{Pt}_{3}$ ), as shown in Fig. 6 and Table 3. The present AXS$\mathrm{RMC}$ results readily demonstrate that for the $[333]_{\mathrm{CN}}$ connection, the $\mathrm{Pt}-\mathrm{Zr}_{3}-\mathrm{Pt}$ linkage is preferred among the common neighbors. These structural features could be attributed to the strong chemical affinity between $\mathrm{Pt}$ and $\mathrm{Zr}$. This is also attributed to the saturation of the quasicrystalline phase in the annealed sample. ${ }^{17}$

The $[211]_{\mathrm{CN}}$ connection can be expressed as edge sharing between two triangles, as shown in Fig. 5. Because the

Table 2 Number, average atomic distance of Pt-Pt root pair, $r$, occupancy of element of the common neighbor atoms of [333 $]_{\mathrm{CN}}$, [322 $]_{\mathrm{CN}}$, and $[211]_{\mathrm{CN}}$ from RMC simulation. The corresponding values for the DRP structure are also presented in round brackets for comparison.

\begin{tabular}{ccccc}
\hline & & & \multicolumn{2}{c}{ Occupancy [\%] } \\
\cline { 4 - 5 } CNA index & Number & $r[\mathrm{~nm}]$ & $\mathrm{Zr}$ & $\mathrm{Pt}$ \\
\hline$[333]_{\mathrm{CN}}$ & $4285(4805)$ & $0.468(0.476)$ & $91.1(82.6)$ & $8.9(17.4)$ \\
{$[322]_{\mathrm{CN}}$} & $913(344)$ & $0.364(0.437)$ & $88.5(86.3)$ & $11.5(13.7)$ \\
{$[211]_{\mathrm{CN}}$} & $6379(5685)$ & $0.509(0.527)$ & $91.1(85.5)$ & $8.9(14.5)$ \\
\hline & $11577(10834)$ & &
\end{tabular}




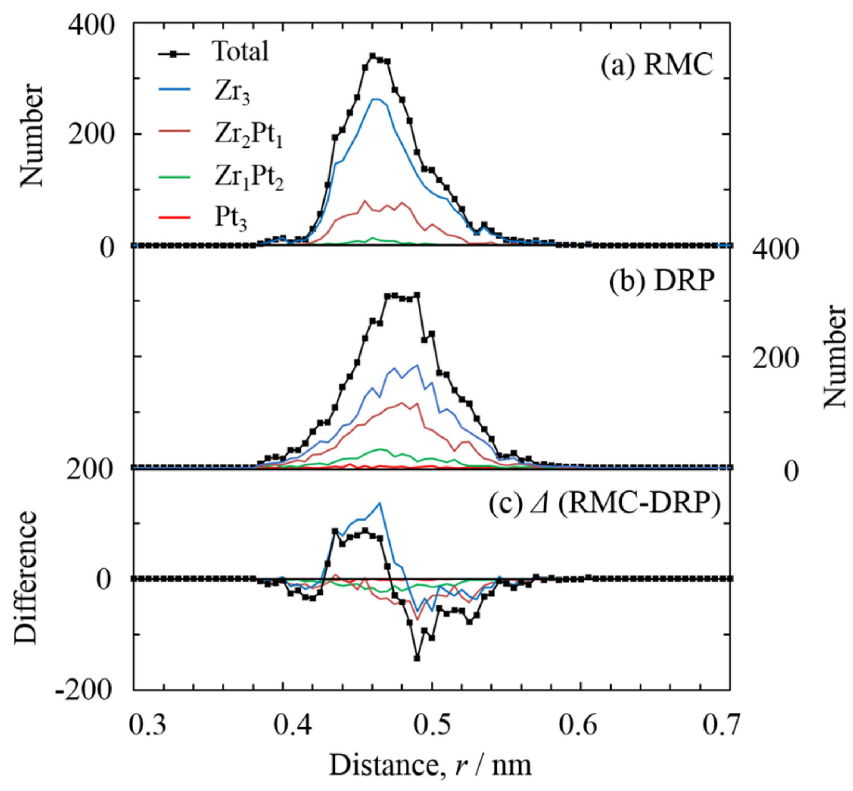

Fig. 6 Distribution of Pt-Pt root pairs with [333] $]_{\mathrm{CN}}$ index for each compositional pattern of common neighbor atoms $\left(\mathrm{Zr}_{3}, \mathrm{Zr}_{2} \mathrm{Pt}, \mathrm{Zr}_{1} \mathrm{Pt}_{2}\right.$, and $\mathrm{Pt}_{3}$ ) from RMC simulation (a), corresponding values for DRP structure (b), and difference between values (c).

Table 3 Number of [333 $]_{\mathrm{CN}}$ indexed Pt-Pt root pairs with each compositional pattern of common neighbors, and average atomic distance, $r$, of Pt-Pt root pairs from RMC simulations. The corresponding values for the DRP structure are also presented in round brackets for comparison.

\begin{tabular}{ccc}
\hline Common neighbor atoms & Number & $r[\mathrm{~nm}]$ \\
\hline $\mathrm{Zr}_{3}$ & $3218(2743)$ & $0.468(0.478)$ \\
$\mathrm{Zr}_{2} \mathrm{Pt}$ & $947(1644)$ & $0.467(0.474)$ \\
$\mathrm{ZrPt}_{2}$ & $93(385)$ & $0.461(0.468)$ \\
$\mathrm{Pt}_{3}$ & $0(33)$ & $-(0.466)$ \\
\hline Total & $4258(4805)$ &
\end{tabular}

$[211]_{\mathrm{CN}}$ connection does not directly lead to a threedimensional packing scheme of atoms, a supplemental bonding chain (SBC) associated with the root-pair atoms is introduced here to discuss the topological image for this connection. The atomic arrangement of common neighbors and SBCs for the $[211]_{\mathrm{CN}}$ connection can be classified as either a linkage of several Bernal polyhedra or an independent polyhedron. As shown in Fig. 7 and Fig. 8, the $[211]_{\mathrm{CN}}$ connections are classified as face-sharing tritetrahedra (FSTT), capped half-octahedron $(\mathrm{CHO})$, partial tetragonal dodecahedra (PTD), partial tri-capped trigonal prism (PTTP), or partial bi-capped tetragonal anti-prism (PBTA), and the independent polyhedron is ranked as a 'non-Bernal polyhedron'.

The AXS-RMC data in Table 4 suggest that the FSTT and $\mathrm{CHO}$ arrangements are preferred in the range of $0.4-0.5 \mathrm{~nm}$, as well as the Zr-rich common neighbors and SBCs. These features are similar to those found for the $[333]_{\mathrm{CN}}$ connection, and are attributed to the $\mathrm{Pt}-\mathrm{Pt}$ middle range ordering associated with the strong bonds in the nearest-neighbor $\mathrm{Pt}-\mathrm{Zr}$ correlation. On the other hand, the PTD, PTTP, PBTA, and non-Bernal polyhedron arrangements predicted from

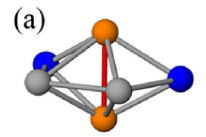

(b)
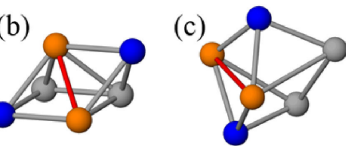

(d-1)

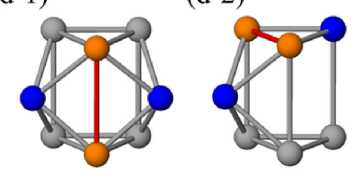

$(\mathrm{e}-1)$
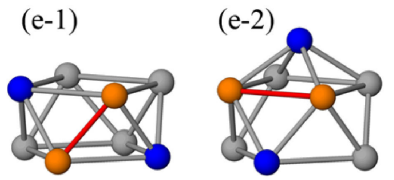

Root pair Common neighbor Supprimental bonding chain

Fig. 7 Schematic diagrams of Bernal polyhedra corresponding to $[211]_{\mathrm{CN}}$, (a) face shared tri-tetrahedra (FSTT), (b) capped half-octahedron (CHO), (c) partial tetragonal dodecahedron (PTD), (d-1,2) partial tri-capped trigonal prism (PTTP), and (e-1,2) partial bi-capped tetragonal anti-prism (PBTA).

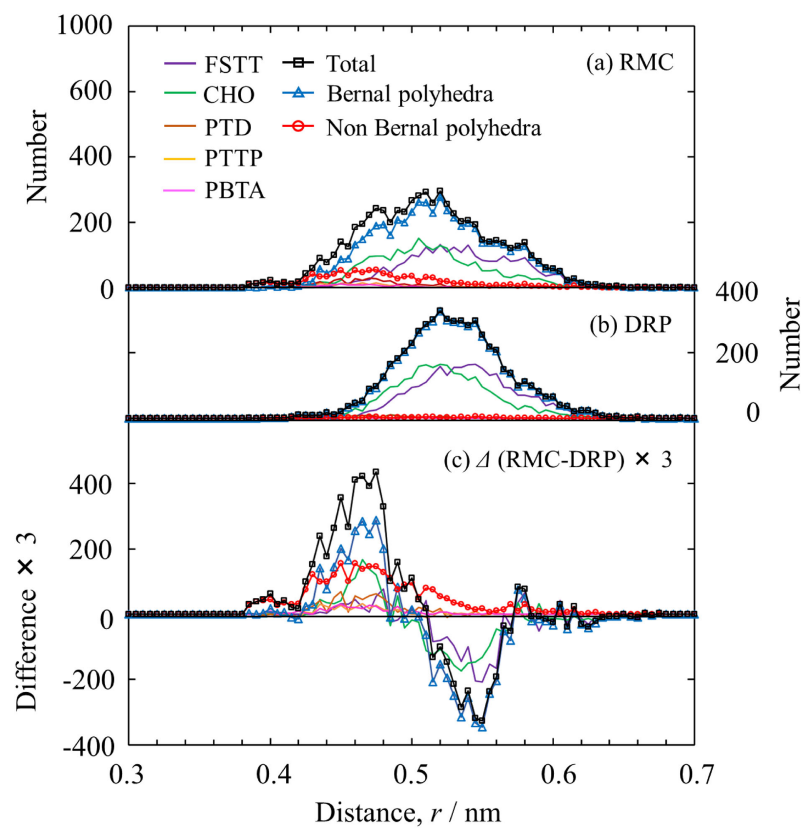

Fig. 8 Distribution of Pt-Pt root pairs with [211 $]_{\mathrm{CN}}$ in middle-range with the corresponding Bernal polyhedra from RMC simulation (a), corresponding values for DRP structure (b), and difference between both values (c). The number of "Bernal polyhedra" is sum the of FSTT, CHO, PTD, PTTP, and PBTA.

the AXS-RMC results indicate a slight preference for $\mathrm{Pt}$ compared to the case of the DRP structure. These structural units with relatively lower packing fractions may differ from the $[333]_{\mathrm{CN}}$ arrangement having a higher packing fraction with $\mathrm{Zr}$-rich common neighbors.

The $[322]_{\mathrm{CN}}$ index corresponds to a structural unit composed of two atoms as the root-pair and three atoms as common neighbors. This $[322]_{\mathrm{CN}}$ structural unit can be expressed as a half octahedron. In order to discuss the threedimensional packing structure associated with Bernal polyhedra, structural analysis with SBC was also employed. The results are illustrated in Figs. 9 and 10 and Table 5. The $[322]_{\mathrm{CN}}$ connections indicate a preference for the PTD, PTTP, PBTA, and non-Bernal polyhedra. Similar to the $[211]_{\mathrm{CN}}$ structural unit, the total amount and fraction of $\mathrm{Pt}$ in the SBCs of these structural units is higher than those of the DRP model. 
Table 4 Numbers, fractions, average Pt-Pt root pair distances, $r$, average composition of common neighbor atoms and SBC atoms for each Bernal polyhedra and non-Bernal polyhedra with [211 $]_{\mathrm{CN}}$ indexed $\mathrm{Pt}-\mathrm{Pt}$ pair.

\begin{tabular}{cccccccc}
\hline & & & & \multicolumn{4}{c}{ Occupancy [\%] } \\
\cline { 5 - 8 } & Count & Fraction [\%] & $r[\mathrm{~nm}]$ & \multicolumn{2}{c}{ Common neighbors } & \multicolumn{2}{c}{ SBCs } \\
& & & & $\mathrm{Zr}$ & $\mathrm{Pt}$ & $\mathrm{Zr}$ & $\mathrm{Pt}$ \\
\hline FSTT & $2436(2784)$ & $38.2(49.0)$ & $0.534(0.538)$ & $95.7(85.7)$ & $4.3(14.3)$ & $83.5(77.7)$ & $16.5(22.3)$ \\
CHO & $2330(2592)$ & $36.5(45.6)$ & $0.509(0.520)$ & $90.8(85.3)$ & $9.2(14.7)$ & $81.8(84.0)$ & $18.2(16.0)$ \\
PTD & $331(136)$ & $5.2(2.4)$ & $0.470(0.478)$ & $86.7(85.3)$ & $13.3(14.7)$ & $77.2(84.9)$ & $22.8(15.1)$ \\
PTTP & $166(72)$ & $2.6(1.3)$ & $0.461(0.465)$ & $89.2(90.3)$ & $10.8(9.7)$ & $77.1(88.1)$ & $22.9(11.9)$ \\
PBTA & $119(19)$ & $1.9(0.3)$ & $0.462(0.467)$ & $87.4(81.6)$ & $12.6(18.4)$ & $71.8(85.5)$ & $28.2(14.5)$ \\
\hline Bernal polyhedra & $5382(5603)$ & $84.4(98.6)$ & $0.513(0.527)$ & $92.7(85.5)$ & $7.3(14.5)$ & $81.6(81.4)$ & $18.4(18.6)$ \\
\hline & & & & & & & \\
\hline Non Bernal polyhedra & $997(82)$ & $15.6(1.4)$ & $0.438(0.510)$ & $82.7(84.8)$ & $17.3(15.2)$ & $74.9(81.7)$ & $25.1(18.3)$ \\
\hline
\end{tabular}

(a)

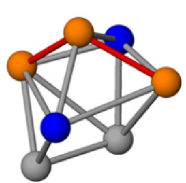

(b)

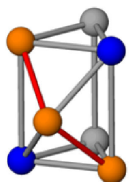

(c)

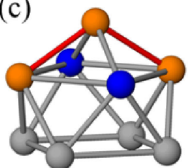

Root pair Common neighbor

Supprimental bonding chain

Fig. 9 Schematic diagrams of Bernal polyhedra corresponding to [322 $]_{\mathrm{CN}}$, (a) partial tetragonal dodecahedra (PTD), (b) partial tri-capped trigonal prism (PTTP), and (c) partial bi-capped tetragonal anti-prism (PBTA).

Based on these structural features, the Pt-Pt middle range correlation associated with the pre-peak can be classified into two types of structures. One structural type is representative of FST, FSTT, and $\mathrm{CHO}$, associated with a higher packing density of atoms and common neighbors. This case also includes SBCs with a strong preference for Zr. The other is represented by PTD, PTTP, PBTA, and non-Bernal polyhedra, having a lower packing density of atoms with common neighbors and SBCs, with slight preference for $\mathrm{Pt}$.

\section{Concluding Remarks}

By using the reverse Monte-Carlo (RMC) simulation method, anomalous X-ray scattering (AXS) data for a $\mathrm{Zr}_{80} \mathrm{Pt}_{20}$ amorphous alloy were analyzed in order to characterize the particular structural features of the alloy, including those associated with the pre-peak signal observed

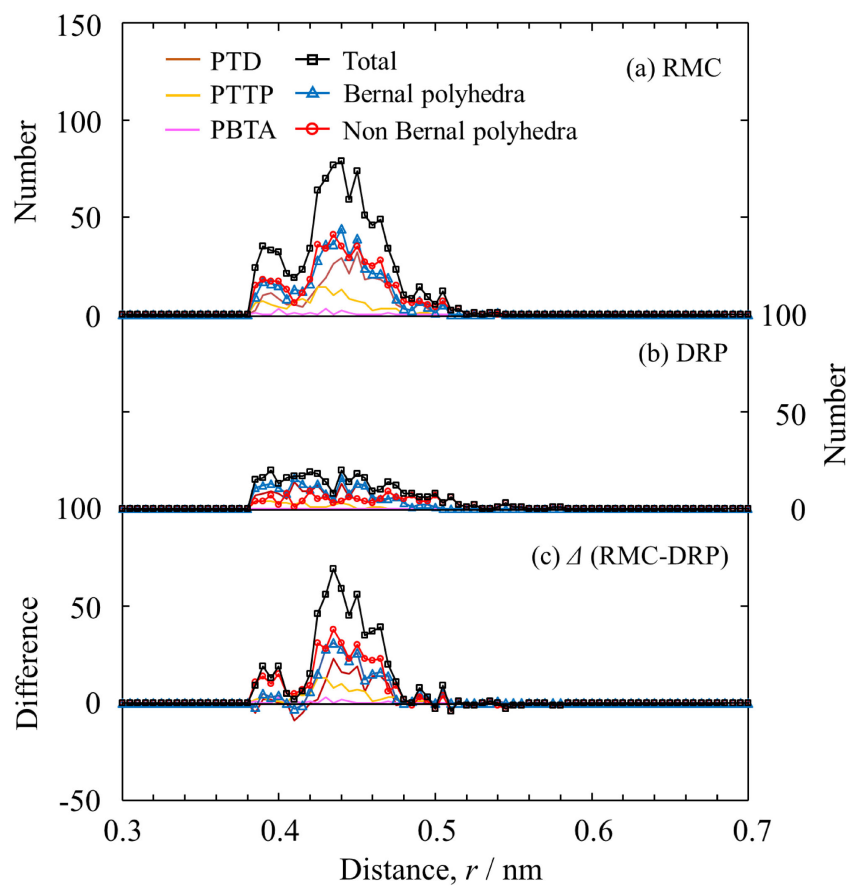

Fig. 10 Pt-Pt root pair distribution with $[322]_{\mathrm{CN}}$ in middle-range with corresponding Bernal polyhedra from RMC simulation (a), corresponding values for DRP structure (b), and difference between both values (c). The number of "Bernal polyhedra" is the sum of PTD, PTTP, and PBTA.

in the X-ray diffraction pattern. The AXS-RMC analysis also provides the $g_{i j}(r) \mathrm{s}$ and $S_{i j}(Q)$ s partial functions for the $\mathrm{Zr}_{80} \mathrm{Pt}_{20}$ amorphous alloy, which are necessary for discussing

Table 5 Numbers, fractions, average Pt-Pt root pair distances, $r$, average composition of common neighbor atoms and SBC atoms of each Bernal polyhedra and non-Bernal polyhedra with $[322]_{\mathrm{CN}}$ indexed $\mathrm{Pt}-\mathrm{Pt}$ pair.

\begin{tabular}{|c|c|c|c|c|c|c|c|}
\hline & \multirow[b]{3}{*}{ Number } & \multirow[b]{3}{*}{ Fraction $[\%]$} & \multirow[b]{3}{*}{$r[\mathrm{~nm}]$} & \multicolumn{4}{|c|}{ Occupancy [\%] } \\
\hline & & & & \multicolumn{2}{|c|}{ Common neighbors } & \multicolumn{2}{|c|}{ SBCs } \\
\hline & & & & $\mathrm{Zr}$ & $\mathrm{Pt}$ & $\mathrm{Zr}$ & $\mathrm{Pt}$ \\
\hline PTD & $291(164)$ & $31.9(47.7)$ & $0.440(0.429)$ & $87.3(88.2)$ & $12.7(11.8)$ & $78.4(83.5)$ & $21.6(16.5)$ \\
\hline РTTP & $131(36)$ & $14.3(10.5)$ & $0.426(0.410)$ & $89.3(82.4)$ & $10.7(17.6)$ & $72.0(79.7)$ & $28.0(20.3)$ \\
\hline PBTA & $13(0)$ & $1.4(-)$ & $0.421(-)$ & $84.6(-)$ & $15.4(-)$ & $71.2(-)$ & $28.8(-)$ \\
\hline Bernal polyhedra & $435(200)$ & $47.6(58.1)$ & $0.435(0.425)$ & $87.8(87.2)$ & $12.2(12.8)$ & $76.8(83.0)$ & $23.2(17.0)$ \\
\hline Non Bernal polyhedra & $478(144)$ & $52.4(41.9)$ & $0.437(0.455)$ & $89.1(85.2)$ & $10.9(14.8)$ & $79.1(81.1)$ & $20.9(18.9)$ \\
\hline
\end{tabular}


the structural features in the middle range ordering. The resultant atomic positions were used to determine the common neighbors, middle range ordering with structural inhomogeneity, and the geometrical features for MRO around a specific atom such as $\mathrm{Pt}$. The main results are summarized as follows:

(1) The present AXS-RMC analysis for the short-range ordering region clearly indicates that the $\mathrm{Pt}$ atom accommodates the coordination ligands of $\mathrm{Zr}$ in the first nearest-neighbor region to form an icosahedral-like local structure.

(2) The information on the partial $S_{P t P t}(Q)$ and the corresponding $g_{P t P t}(r)$ clearly suggests that the prepeak signal at $17 \mathrm{~nm}^{-1}$ in $S(Q)$ is mainly attributed to the $\mathrm{Pt}-\mathrm{Pt}$ middle range correlation at approximately $0.4-0.5 \mathrm{~nm}$. This particular middle range ordering of Pt-Pt correlations corresponds mainly to the polyhedral connections described by the $[333]_{\mathrm{CN}},[211]_{\mathrm{CN}}$, and $[322]_{\mathrm{CN}}$ indices.

(3) The geometrical and chemical features of the common neighbors of the middle-range $\mathrm{Pt}-\mathrm{Pt}$ pairs indicate unique CSRO and TSRO with both chemical and density fluctuations in two distinct regions: the Zr-rich region with high packing density and the Pt-rich region with low packing density.

(4) Notably, in this particular middle range ordering of $\mathrm{Pt}-\mathrm{Pt}$ correlations, chemical and density fluctuations were both clearly identified by using the unique geometrical linkages based on the Bernal polyhedral units. This new approach is expected to be utilized for characterizing the middle range ordering of a variety of amorphous alloy systems.

\section{Acknowledgment}

The authors express their gratitude to Dr. H. Nitani, Institute of Materials Structure Science, High Energy Accelerator Research Organization, Tsukuba, Japan, for assistance with the AXS measurements (Proposal No. 2019G646).

We are also grateful to Professor Y. Waseda, Tohoku University for his valuable comments and discussions. A part of this work was supported by the Japan Society for the Promotion of Science (JSPS) KAKENHI Grant Numbers JP16H04210 and JP19K15653.

\section{REFERENCES}

1) M.F. Ashby and A.L. Greer: Scr. Mater. 54 (2006) 321-326.

2) C.A. Schuh, T.C. Hufnagel and U. Ramamurty: Acta Mater. 55 (2007) 4067-4109.

3) M.M. Trexler and N.N. Thadhani: Prog. Mater. Sci. 55 (2010) 759839.

4) A. Inoue and A. Takeuchi: Acta Mater. 59 (2011) 2243-2267.

5) T. Kawamata, Y. Yokoyama, M. Saito, K. Sugiyama and Y. Waseda: Mater. Trans. 51 (2010) 1796-1801.

6) T. Fukunaga, K. Itoh, T. Otomo, K. Mori, M. Sugiyama, H. Kato, M. Hasegawa, A. Hirata, Y. Hirotsu and A.C. Hannon: Mater. Trans. 48 (2007) 1698-1702.

7) H.W. Sheng, W.K. Luo, F.M. Alamgir, J.M. Bai and E. Ma: Nature 439 (2006) 419-425.

8) K. Saksl, P. Jóvári, H. Franz and J.Z. Jiang: J. Appl. Phys. 97 (2005) 113507.

9) K. Saksl, P. Jóvári, H. Franz, Q.S. Zeng, J.F. Liu and J.Z. Jiang: J. Phys. Condens. Matter 18 (2006) 7579-7592.

10) S.Y. Wang, C.Z. Wang, M.Z. Li, L. Huang, R.T. Ott, M.J. Kramer, D.J. Sordelet and K.M. Ho: Phys. Rev. B 78 (2008) 184204.

11) L. Yang, J.Z. Jiang, K. Saksl and H. Franz: J. Phys. Condens. Matter 19 (2007) 476217.

12) J. Saida, M. Matsushita and A. Inoue: J. Appl. Phys. 90 (2001) 47174724.

13) B.S. Murty, D.H. Ping, M. Ohnuma and K. Hono: Acta Mater. 49 (2001) 3453-3462.

14) D.J. Sordelet, R.T. Ott, M.Z. Li, S.Y. Wang, C.Z. Wang, M.F. Besser, A.C.Y. Liu and M.J. Kramer: Metall. Mater. Trans. A 39 (2008) 19081916.

15) N.A. Mauro, V. Wessels, J.C. Bendert, S. Klein, A.K. Gangopadhyay, M.J. Kramer, S.G. Hao, G.E. Rustan, A. Kreyssig, A.I. Goldman and K.F. Kelton: Phys. Rev. B 83 (2011) 184109

16) A. Hirata, L.J. Kang, T. Fujita, B. Klumov, K. Matsue, M. Kotani, A.R. Yavari and M.W. Chen: Science 341 (2013) 376-379.

17) K. Sugiyama, T. Kawamata and T. Muto: J. Phys. Conf. Ser. 809 (2017) 012005 .

18) Y. Waseda, E. Matsubara and K. Sugiyama: Sci. Rep. Res. Inst. Tohoku Univ. A 34 (1988) 1-14.

19) M. Saito, C. Park, K. Omote, K. Sugiyama and Y. Waseda: J. Phys Soc. Jpn. 66 (1997) 633-640.

20) D.T. Cromer and D. Liberman: J. Chem. Phys. 53 (1970) 1891-1898.

21) Y. Waseda: The Structure of Non-Crystalline Materials, (McGraw-Hill International, New York, 1980) pp. 27-51.

22) R.L. McGreevy and L. Pusztai: Mol. Simul. 1 (1988) 359-367.

23) A.S. Clarke and J.D. Wiley: Phys. Rev. B 35 (1987) 7350-7356.

24) E.A. Brandes and G.B. Brook: Smithells Metals Reference Book, 7th ed., (Elsevier Butterworth-Heinemann Ltd., London, 1992) pp. 4-41.

25) A. Takeuchi and A. Inoue: Mater. Trans. 46 (2005) 2817-2829.

26) J.D. Bernal: Nature 183 (1959) 141-147.

27) A.S. Clarke and H. Josson: Phys. Rev. E 47 (1993) 3975-3984.

28) J.D. Honeycutt and H.C. Andersen: J. Phys. Chem. 91 (1987) 49504963.

29) J.D. Bernal: Nature 185 (1960) 68-70. 